\title{
Collection Efficiency of the Twin EDS Detectors for Quantitative X-ray Analysis on A New Probe-Corrected TEM/STEM
}

\author{
Jane Y. Howe ${ }^{1}$, Tarunika Ramprasad ${ }^{2}$, Akinari Hanawa ${ }^{3}$, Hiromi Inada ${ }^{3}$, Jose Jimenez ${ }^{1}$, David Hoyle ${ }^{4}$, \\ Edgar Voelkl ${ }^{1}$, and Thomas J. Zega ${ }^{2,5}$ \\ 1. Hitachi High-Technologies America Inc., Clarksburg, USA. \\ 2. Department of Materials Science and Engineering, University of Arizona, USA. \\ 3. Hitachi High Technologies Co., Hitachinaka, Japan. \\ 4. Hitachi High Technologies Canada Inc., Toronto, Canada. \\ 5. Lunar and Planetary Laboratory, University of Arizona, USA.
}

This paper reports a systematic study of the collection efficiency of an X-ray energy dispersive spectrometer (EDS) equipped on a newly installed $200 \mathrm{kV}$ TEM/STEM. Understanding of the collection efficiency is critical to quantitative compositional measurements, and several factors affect the overall collection efficiency. Solid angle is arguably the most important parameter, which describes the angular extent of X-rays emitted by a point source and collected by the detector system [1-2]. Once a particular EDS system is installed on a specific TEM, the solid angle remains a constant. However, there are several variables that affect the performance, such as the specimen holder penumbra (shadowing), specimen eucentric height, probe current, accelerating voltage, and number and location of the detectors used (in the case of multiple-detector configuration).

Herein, we evaluate the collection efficiency of the twin Oxford X-Max 100TLE EDS system on the probe-corrected Hitachi HF5000 TEM/STEM at the University of Arizona. Shown in Figure 1, two rectangular-shaped $100 \mathrm{~mm}^{2}$ window-less X-Max 100TLE detectors are oriented normal to the axis of the specimen holder, $180^{\circ}$ apart. Following Zaluzec formula [2], the solid angle of each detector is calculated to be $0.85 \mathrm{sr}$, providing a total angle of $1.70 \mathrm{sr}$ for the twin configuration. We measured the penumbra of the Gatan 646 double-tilt low-background $(\mathrm{Be})$ holder using the EDS "Test specimen" recommended by Zaluzec [3]. The Test Specimen is a $20 \mathrm{~nm} \mathrm{Ge} / 20 \mathrm{~nm} \mathrm{SiN} / 20 \mathrm{~nm}$ thick membrane $(500 \mu \mathrm{m} \times 500 \mu \mathrm{m})$ with arrays of $2 \mu \mathrm{m}$-diameter holes on a Si wafer (TEMwindows/SiMPore Inc.) [3]. With the known thickness, using N K, Si K, Ge K and L peaks, this specimen can be used to calibrate the energy resolution over a wide range. It can also be used to monitor the possible deterioration due to detector contamination over time. We undertook all the measurements at $200 \mathrm{kV}$ at a probe current of $1.40 \mathrm{nA}$ unless otherwise stated.

Fig. 2 plots the Ge $\mathrm{K} \alpha$ peak intensity as a function of the $\alpha$-tilt from $-10^{\circ}$ to $+10^{\circ}$ while using Detector 1 alone (positive tilt is in the clockwise direction towards Detector 1) and in twin configuration. It shows that using only Detector 1 , the penumbra decreased linearly with increasing $\alpha$ tilt. The counts almost doubled when tilted from -10 to $+10^{\circ}$. However, the penumbra profile in the twin-detector setting is much flatter throughout out the range, with less than $10 \%$ intensity variation. We are going to further investigate the penumbra in the single and twin-configuration as a function of the full $\alpha$ and $\beta$ tilt range. We also acquired EDS measurements at various probe currents. Fig. 3 presents the Ge $\mathrm{K} \alpha$ peak intensity as a function of the probe current. The X-ray counts increased linearly with the probe current except the first data point. We will repeat the experiment and verify whether it was a measuring error. In summary, the findings from this study will enable us to optimize the experimental setup for quantitative X-ray analysis at its maximal collection efficiency [4]. 


\section{References:}

[1] NJ Zaluzec, Microsc Microanal (2013) 19 (S2) 1262.

[2] NJ Zaluzec, Microsc Microanal (2014) 201318.

[3] NJ Zaluzec, JP DesOrmeaux, and J Roussie, Microsc Microanal (2016) 22(S3) 322.

[4] Research supported by NSF grant number 1531243 and NASA grant numbers NNX15AJ22G and NNX12AL47G. The work was carried out at the Kuiper Core Imaging and Microscopy Facility, University of Arizona. We thank Dr. Nestor Zaluzec for the insightful discussions.
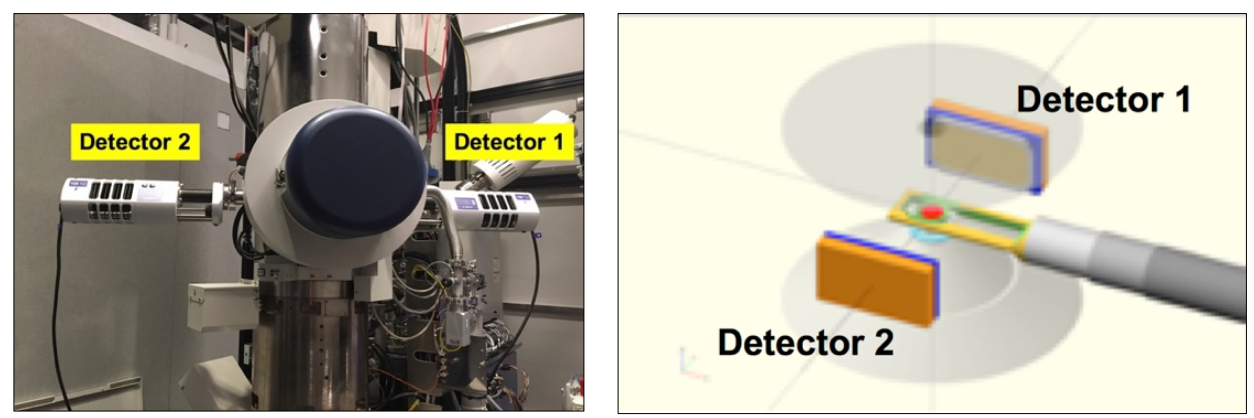

Fig. 1. A photo and a drawing that show the configuration of the twin Oxford X-Max 100TLE EDS system on the Hitachi HF-5000 TEM/STEM.

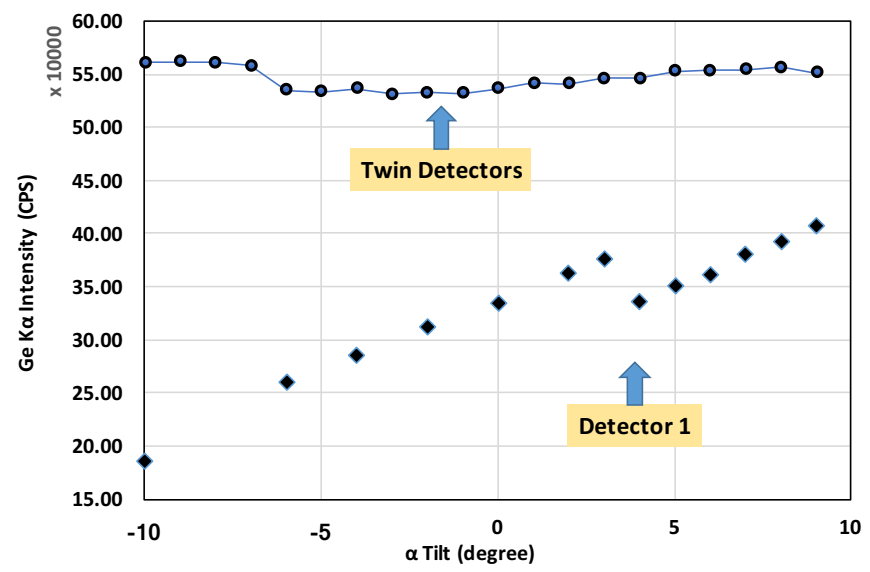

Fig. 2. Comparison of the penumbra using only Detector 1 and twin-detector configurations. Probe current and sample area were the same in both experiments.

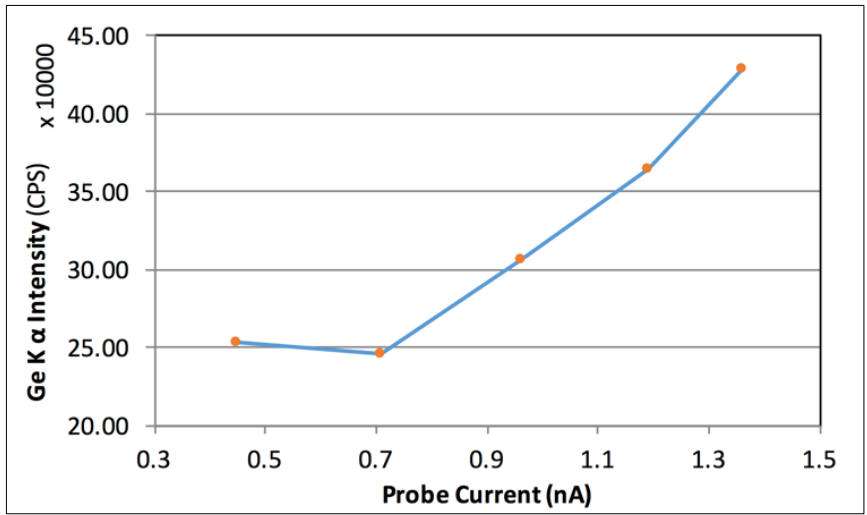

Fig. 3. Ge Ka intensity as a function of probe current at $200 \mathrm{kV}$. 